\title{
Embedded protocols based on the crowd Petri networks and opportunistic bandwidth allocation
}

\author{
Shu-yi Guo* and Qi Si
}

\begin{abstract}
In order to improve the bandwidth utilization of embedded system and the working efficiency of mobile system, we propose a crowd Petri network and bandwidth allocation scheme. These research results are suitable for mobile embedded system. On the one hand, we have established a mobile crowd network system based on crowd Petri net. The system can give full play to the advantages of the concurrent and distributed data, so as to provide the formal description of the data control behavior of the mobile system and the asynchronous concurrent protection of the mobile service. On the other hand, through the opportunistic bandwidth allocation, the system efficiency and the network resources of the crowd Petri network is the most appropriate configuration. In the process of optimizing the crowd data, an embedded control protocol is studied based on the combination of the user demand and the data element characteristics by the combination of the service quality and the resource consumption. Simulation results show the effectiveness and feasibility of the embedded protocol based on bandwidth allocation of crowd Petri network.
\end{abstract}

Keywords: Embedded system, Crowd Petri networks, Opportunistic control, Bandwidth allocation

\section{Introduction}

In the mobile network, how to balance the [1] and service satisfaction [2] performance for providing an inevitable trend [3] for embedded users and high efficiency reliable service [4] has become an inevitable trend. However, the dynamic topology, embedded redundancy, and embedded user dynamic demand of mobile embedded network [5] makes the limited network resources cannot satisfy the requirements of embedded users.

The Joint Optimization Methods was proposed in [6], which is able to solve the non-convex problems and provides a general solution to the resource allocation for relay enhanced multi-carrier systems. Aristomenopoulos $G$ et al. [7] studied the efficient-distributed resource allocation and users to cells assignment over a heterogeneous integrated wireless environment. The particular game equation and its corresponding notion of equilibrium were studied by Perlaza S M et al. [8]. The paper [9] proposed a model of the connectivity of a wireless

\footnotetext{
* Correspondence: shyiguo@sina.com

North China University of Water Resources and Electric Power, Zhengzhou 450011, China
}

sensor network deployed over a square area, which considers border effects. Du Z et al. [10] proposed an online network selection algorithm to learn the optimal network selection policy with network handoff cost consideration.

The first time the concept of transition covers in Petri net models was proposed for obtaining a live Petri net controller of small size [11]. The authors of article [12] proposed a new methodology to design and analyze an urban traffic network control system by using the STPN (synchronized timed Petri nets), which also modularized the applications of the STPN to eight-phase, six-phase, and two-phase traffic-light control systems. A Petri netbased method was presented to address the temporal constraint satisfiability in a holistic manner and also in a modular way in article [13]. The automatic Web service composition method was presented by the authors of article [14] that deals with both input/output compatibility and behavioral constraint compatibility of fuzzy semantic services. The admissible heuristic function was designed in article [15] which considered the available time of shared machine resources and subparts during calculating 
Table 1 Descriptions of EM

\begin{tabular}{ll}
\hline Parameters & Content \\
\hline NODE_S & Sending node \\
B_size & Crowd data buffer size of embedded node \\
NEXT_data & The next sending data objects \\
Crowd_N & The maximum crowd data size \\
\hline
\end{tabular}

the lower bound of the remaining time for unprocessed operations. The authors of article [16] presented a method for hierarchical configurable Petri nets description in VHDL language.

Based on the above research results such as Joint Optimization [6], efficient distributed resource allocation [7], online network selection algorithm [9], and Transition cover-based design of Petri net controllers [11], combined with our previous research results [17], we proposed the embedded protocols based on the crowd Petri networks with the opportunistic bandwidth allocation.

The rest of the paper is organized as follows. Section 2 describes the Crowd Petri Networks System. Section 3 gives the embedded protocols based on opportunistic bandwidth allocation. The experimental results are shown in Section 4. Finally, Section 5 concludes this paper.

\section{Crowd Petri Networks System}

Based on the characteristics of mobile communication network system, transmission control, and embedded service, we have established a mobile crowd network system based on Petri network. This system has the characteristics of service concurrency and distributed data. These features can provide the formal description of the data control behavior and the asynchronous concurrent protection for the mobile system.

Crowd Petri net is a combination of the virtual advantages of the original Petri net and the mobile crowd service. Crowd Petri network can play the advantages of Petri network graphics simulation of complex systems based on the mobile crowd requirements. Crowd Petri net system model consists of three types of elements. The first type element represents the moving

Table 2 Descriptions of $C R$ and $M C$

\begin{tabular}{ll}
\hline Parameters & Content \\
\hline CR_node & Crowd service random users \\
MC_node & $\begin{array}{l}\text { Data element node for transmission control } \\
\text { of mobile communication }\end{array}$ \\
CR_d_seq & Random user data sequence of crowd service \\
MC_d_seq & Mobile communication feedback data sequence \\
Crowd_q_len & crowd data queue length \\
RC_buf & Reconstruction data buffer \\
Crowd_fusion & Degree of fusion of crowd Petri networks \\
\hline
\end{tabular}

Description: Control algorithm of crowd Petri net. Input: RC_buf, $\mathrm{MC}$ d_seq, CR_d_seq;

Algorithm: cpnc(MC_Node, $C \bar{R} \_\bar{N}$ Node $)$

void InitSeq()

\{

$\mathrm{n}=$ length(CR_buf);

$\mathrm{m}=$ length $\left(\mathrm{MC} \overline{\mathrm{C}}_{-} \mathrm{d}\right.$ seq $)$;

$\mathrm{q}=$ length $\left(\mathrm{CR} \_\mathrm{d} \_\right.$seq);

\}

void AssignSeq()

\{

content $(\mathrm{CR}$ _buf $)=$ content $\left(\mathrm{CR} \_\right.$Node $)$;

content $\left(\mathrm{MC} \mathrm{C}_{-}\right.$_seq $)=\operatorname{content}\left(\overline{\mathrm{M}} \mathrm{C}_{-}\right.$Node $)$;

content $\left(\mathrm{CR} \_\right.$___ $\_$seq $)=$content $\left(\mathrm{CR} \_\overline{\text { Node}}\right)$;

\}

Void Number_Node()

\{

$\mathrm{nCR}=$ number(CR Node);

$\mathrm{nMC}=$ number( $\mathrm{MC}$ _ Node $)$;

\}

main()

\{

InitSeq();

AssignSeq();

If $(\mathrm{nCR}>\mathrm{nMC})$

else

control(CR_Node);

If( $(n>m)$

control(MC_Node);

control(RC_buf);

Else if $(m>q)$

control(MC_d_seq);

else

control(CR_d_seq);

\}

state of the embedded node. The second element indicates the random variation of crowd service. The third type of element represents the transmission control state of the mobile communication.

Three state elements of crowd Petri network is denoted as $E M_{-}$element, $\mathrm{CR}_{-}$element, and $\mathrm{MC}_{-}$element. The EM_ elements, $\mathrm{CR}_{-}$elements, and $\mathrm{MC}_{-}$elements would be analyzed and updated with the equal probability in crowd Petri network. EM_ element is driven by CR_ elements. $\mathrm{CR}_{-}$element is described by $\mathrm{MC}_{-}$element. CR_ elements are obtained by the integration of EM_ and MC_ elements.

Crowd Petri network is a crowd reconstruction of mobile communication embedded data elements. This reconstruction effectively simplifies the data type and data competition of Petri net. The formal definition of crowd Petri net is as follows: 


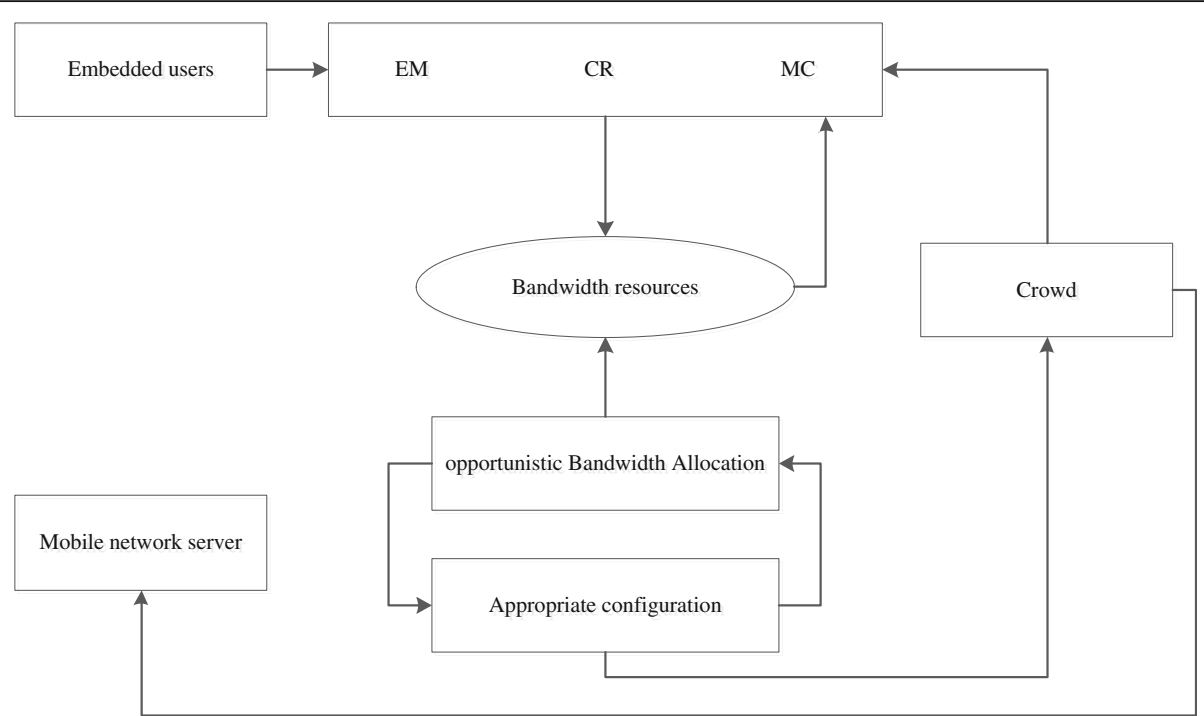

Fig. 1 Crowd Petri net transfer rule

Crowd Petri net has to satisfy the following conditions:

(1) Mobile crowd data element collection cannot be empty.

(2) The location set of EM is the mobile location information of the mobile node in the embedded state.

The state transition set of $\mathrm{CR}$ and the finite data element set of MC have to satisfy the relation shown as Eq. (1).

$$
\left\{\begin{array}{l}
L(E M) \cap T(C R)=\varnothing \\
D(M C) \cap T(C R) \subseteq L(E M)
\end{array}\right.
$$

Crowd Petri net can reasonably release the mobile embedded system user data to other users of the system with the equal probability. The parameters of EM_ element, CR_ element, and $M C_{-}$elements are described in Tables 1 and 2.

The algorithm of crowd Petri net control is shown as follows:

Here, function of control is completed according to Fig. 1. Function of number is used to record the length of data sequence. Function of number is used to analyze the number of nodes.

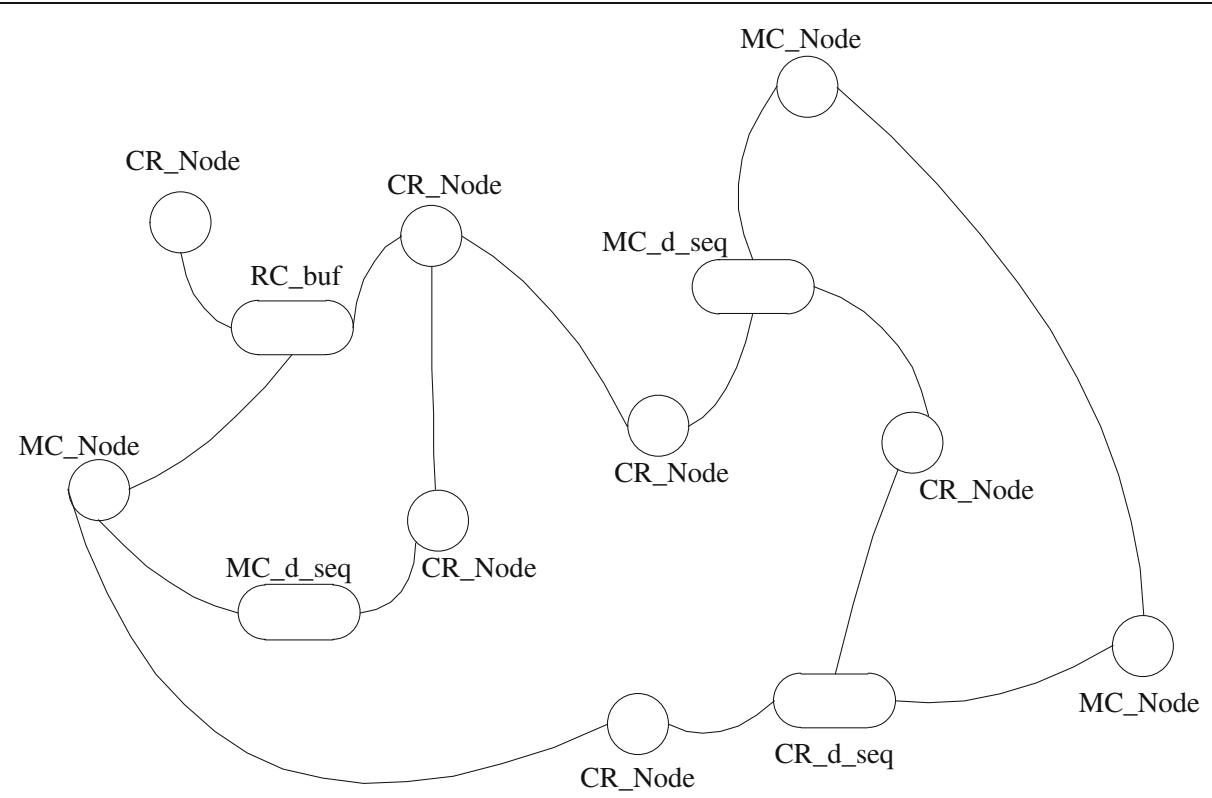

Fig. 2 Crowd structure model for mobile networks 


\section{Embedded protocols based on opportunistic bandwidth allocation}

Introducing the opportunistic bandwidth allocation in the crowd Petri network system is helpful to the decision of the mobile users and the control of the resources. By opportunistic bandwidth allocation, the system efficiency and network resources of the crowd Petri network are the most appropriate configuration. In the process of optimizing the crowd data, a kind of embedded control protocol is studied based on the combination of the user requirements and the data element characteristics, by the combination of the service quality and the resource consumption.

Based on crowd Petri net and opportunistic scheduling, we take the bandwidth resources as the opportunistic metadata, which take opportunistic embedded user and mobile network services, respectively, as the demand side and the security side of the bandwidth. The crowd framework model is shown in Fig. 2.

The probability of setting the opportunity is $\mathrm{P}_{\mathrm{C}}$. The bandwidth of the embedded user is $B_{R}$. the appropriate bandwidth resources would be allocated to embedded users by opportunity allocation utility function $\mathrm{O}_{\mathrm{A}}\left(\mathrm{P}_{\mathrm{C}}\right)$. The function must have the following properties:

$(1) \mathrm{O}_{\mathrm{A}}\left(\mathrm{P}_{\mathrm{C})}\right.$ is monotonic linear.

(2) $\mathrm{O}_{A}\left(\mathrm{P}_{C}\right)$ is monotonically increasing or decreasing.

(3) There is a linear mapping between $\mathrm{B}_{R}(\mathrm{PC})$ and $\mathrm{O}_{\mathrm{A}}$.

Embedded users want to maximize the utilization of bandwidth resources. The optimal allocation of bandwidth resources in embedded protocols is demonstrated as Eq. (2).

$$
\mathrm{B}_{\mathrm{R}}==\sum_{i=1}^{K} \mathrm{EM}_{i} \times \mathrm{MC}(i)-\arg \left(\mathrm{O}_{\mathrm{A}}\left(\mathrm{P}_{\mathrm{C}}\right)\right)
$$

Then, according to Eq. (3), the optimal crowd mobile network and the appropriate adjustment of the mobile embedded bandwidth resources are optimized. The proportion of users with the maximum appropriate bandwidth is $A B_{E U}$, which is provided by the embedded users.

$$
\mathrm{AB}_{\mathrm{EU}}=\frac{\sum_{i=1^{N(\mathrm{AB})}} \mathrm{B}_{\mathrm{R}}(i)}{\sum \mathrm{EU}}
$$

Eqs. (2) and (3) can design a new type of embedded control protocol. The embedded protocol is suitable for complex systems and dynamic user needs.
Table 3 Comparison of bandwidth allocation

\begin{tabular}{lll}
\hline Performance metric & $\begin{array}{l}\text { Performance } \\
\text { of EP-CPOBA }\end{array}$ & $\begin{array}{l}\text { Performance } \\
\text { of EP-ALONE }\end{array}$ \\
\hline Opportunistic weight & 0.2 & 0 \\
Max users & 5 & 2 \\
Resource utilization ratio (\%) & 100 & 45 \\
Moderate resource satisfaction (\%) & 100 & 35 \\
\hline
\end{tabular}

\section{Experiment results}

Simulation results show the effectiveness and feasibility of the proposed embedded protocol denoted as EPCPOBA. Experiment compared the performance of the proposed EP-CPOBA with a single embedded algorithm denoted as EP-ALONE. The performance evaluation would demonstrate improvement of the average satisfaction of users and the system revenue with the proposed EP-CPOBA. Mobile network topology range is $1200 \mathrm{~m} \times$ $1000 \mathrm{~m}$. The bandwidth capacity is $1 \mathrm{Mbps}$. The concurrent user number value is from 1 to 5 . Each embedded user moderate bandwidth resource demand $\mathrm{R}_{\mathrm{AB}}$ would be selected randomly in $10-50 \%$. Simulation time is $2300 \mathrm{~s}$.

Table 3 gives the comparison results of the above algorithms. The maximum number of concurrent users supported by the EP-CPOBA mechanism is five, which is significantly higher than that of EP-ALONE. About the embedded user appropriate resource satisfaction and channel utilization, EP-CPOBA is significantly higher than that of EP-ALONE. The appropriate resource satisfaction of embedded users of the EP-CPOBA in mobile system is higher than that of single embedded protocol.

Figures 3 and 4 give the change trend of the resource utilization of embedded users with the number of concurrent users. It can be seen that the resource utilization of embedded users will be affected by some external

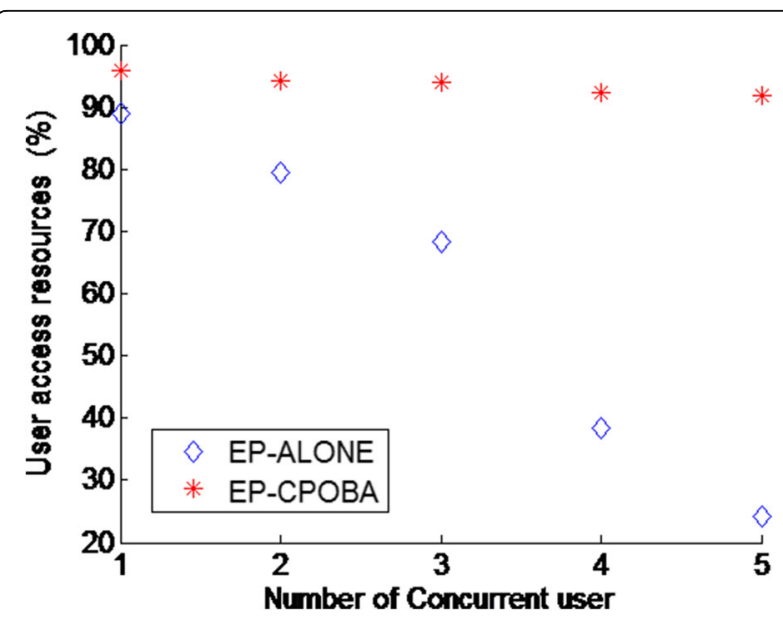

Fig. 3 User access resources with is $\mathrm{R}_{A B} 10 \%$ 


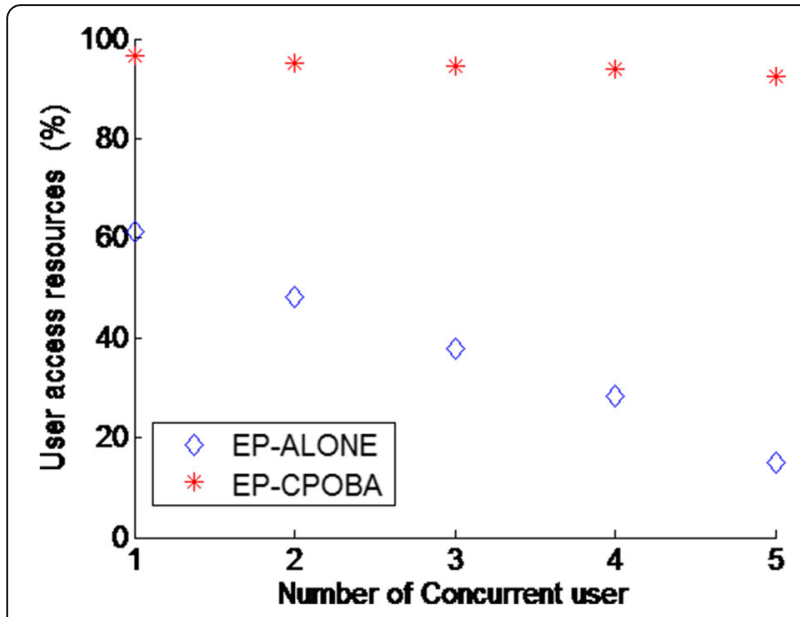

Fig. 4 User access resources with is $\mathrm{R}_{A B} 20 \%$

factors, there will be some ups and downs. The overall jitter of the EP-CPOBA is small. In comparison of Figs. 3 and 4, we can see that EP-ALONE is difficult to satisfy the requirements of high bandwidth resources. However, at the beginning of a short period of time, the embedded user resource utilization rate of the EP-CPOBA is higher than the EP-ALONE by only 0.1 percentage points. In constant opportunistic scheduling and embedded sensing, by searching the resource allocation and cycle optimization, the EPCPOBA users can allocate resources to achieve a balance. The mobile network status is stable as shown in Figs. 5 and 6.

\section{Conclusions}

We proposed a crowd Petri net and bandwidth opportunistic allocation scheme, which are suitable for mobile embedded systems. The system can improve the bandwidth

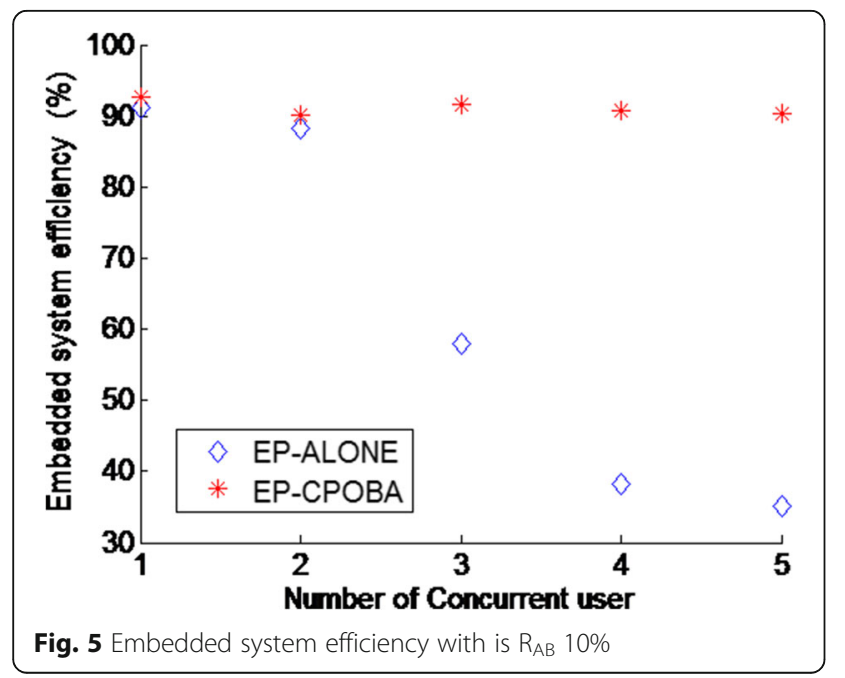

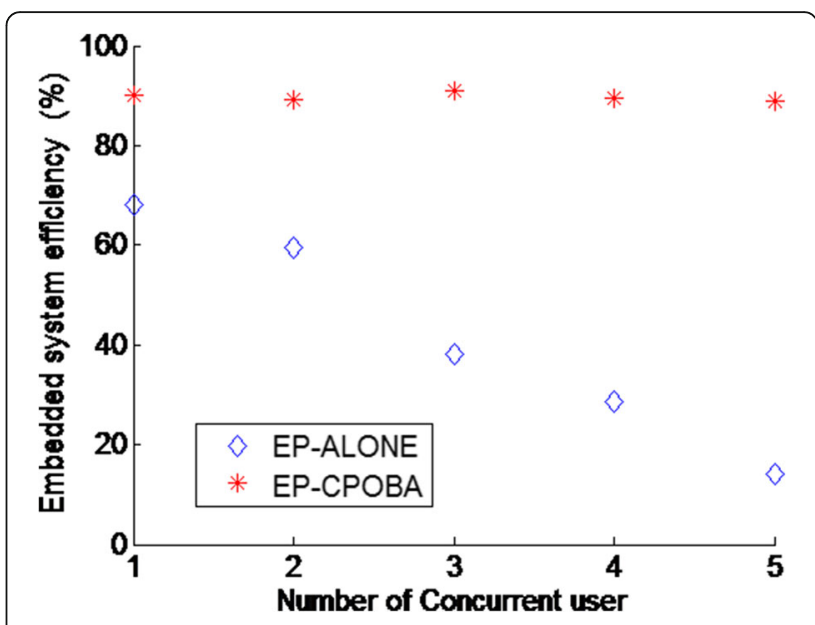

Fig. 6 Embedded system efficiency with is $R_{A B} 20 \%$

utilization rate of embedded system and the working efficiency of the mobile system. First of all, the crowd Petri net was designed. Then the mobile crowd network system is proposed. We studied the formal description of the behavior of the data control and the asynchronous concurrency of the mobile service, which make the system have the advantage of service concurrency and distributed data. Second, the opportunistic bandwidth resource allocation scheme was established. This scheme can further improve the system efficiency and network resource allocation of Petri net. Finally, an embedded control protocol is proposed based on the user requirements and data element characteristics. Simulation experiments compared the bandwidth of the embedded protocol and a single embedded protocol based on the bandwidth allocation of Petri net. The results show that the proposed embedded protocol always has the highest utilization of user resources, embedded user satisfaction, and system efficiency.

Authors' contributions

The structuration and coding part was carried out by SG, and the testing and debugging part was done by both the authors. This manuscript had been prepared and checked by both of the authors together. Both authors read and approved the final manuscript.

\section{Competing interests}

The authors declare that they have no competing interests.

Received: 23 August 2016 Accepted: 27 December 2016 Published online: 14 January 2017

\section{References}

1. Y Song, Y Sun, W Shi, A two-tiered on-demand resource allocation mechanism for vm-based data centers. IEEE Trans. Serv. Comput. 6(1), 116-129 (2013)

2. Q Han, N Venkatasubramanian, Timeliness-accuracy balanced collection of dynamic context data. IEEE Trans. Parallel Distrib. Syst. 18(2), 158-171 (2007)

3. C Li, L Li, Joint optimization of resource allocation and user QoS satisfaction control for efficient grid resource management and scheduling. IEEE Syst. J. 3(1), 65-77 (2009) 
4. JH Chow, RW De Mello, KW Cheung, Electricity market design: an integrated approach to reliability assurance. Proc. IEEE 93(11), 1956-1969 (2005)

5. B San Miguel, JM Del Alamo, JC Yelmo, A personal data framework for distributed user modelling in new service ecosystems. IEEE Lat. Am. Trans. 13(11), 3604-3611 (2015)

6. Y Fu, Q Zhu. Joint Optimization Methods for Nonconvex Resource Allocation Problems of Decode-and-Forward Relay-Based OFDM Networks[]]. IEEE Trans. Veh. Technol. 65(7),1-1 (2016)

7. G Aristomenopoulos, T Kastrinogiannis, S Papavassiliou, Multiaccess multicell distributed resource management framework in heterogeneous wireless networks. IEEE Trans. Veh. Technol. 61(6), 2636-2650 (2012)

8. SM Perlaza, $\mathrm{H}$ Tembine, S Lasaulce et al., Quality-of-service provisioning in decentralized networks: a satisfaction equilibrium approach. IEEE J. Sel. Top. Sign. Proces. 6(2), 104-116 (2012)

9. L Laranjeira, G Nunes Rodrigues, Border effect analysis for reliability assurance and continuous connectivity of wireless sensor networks in the presence of sensor failures. IEEE Trans. Wirel. Commun. 13(8), 4232-4246 (2014)

10. Z Du, Q Wu, P Yang, Dynamic user demand driven online network selection. IEEE Commun. Lett. 18(3), 419-422 (2014)

11. H Liu, K Xing, MC Zhou et al., Transition cover-based design of Petri net controllers for automated manufacturing systems. IEEE Trans. Syst. Man Cybern. Syst. Hum. 44(2), 196-208 (2014)

12. YS Huang, YS Weng, MC Zhou, Modular design of urban traffic-light control systems based on synchronized timed Petri nets. IEEE Trans. Intell. Transp. Syst. 15(15), 530-539 (2014)

13. Y Du, W Tan, MC Zhou, Timed compatibility analysis of web service composition: a modular approach based on Petri nets. IEEE Trans. Autom. Sci. Eng. 11(2), 594-606 (2014)

14. J Cheng, C Liu, MC Zhou et al., Automatic composition of semantic web services based on fuzzy predicate petri nets. IEEE Trans. Autom. Sci. Eng. 12(2), 680-689 (2015)

15. $\mathrm{C} L i, \mathrm{~W} W \mathrm{~W}, \mathrm{Y}$ Feng et al., Scheduling FMS problems with heuristic search function and transition-timed Petri nets. J. Intell. Manuf. 26(5), 1-12 (2015)

16. M Doligalski, M Adamski, Hierarchical configurable Petri net modeling in VHDL. International Journal of Electronics and Telecommunications 58(4), 397-402 (2016)

17. S Guo, Q Si, Mechanical hydraulic characteristic analysis scheme based on lightweight crowd data in mobile embedded devices. J Embed. Syst. 2017, 13 (2017). doi:10.1186/s13639-016-0049-3

\section{Submit your manuscript to a SpringerOpen ${ }^{\mathcal{O}}$ journal and benefit from:}

- Convenient online submission

$\checkmark$ Rigorous peer review

- Immediate publication on acceptance

- Open access: articles freely available online

- High visibility within the field

- Retaining the copyright to your article 